\title{
Surgical techniques, oncologic and functional outcomes of two types of modified ileal orthotopic neobladders
}

\author{
Qinxin Zhao ${ }^{1 \#}$, Feiya Yang ${ }^{1 \#}$, Han $\mathrm{Hao}^{2 \#}$, Xinfei $\mathrm{Li}^{2 \#}$, Liyuan $\mathrm{Wu}^{1}$, Xuesong $\mathrm{Li}^{2}$, Nianzeng Xing ${ }^{1}$ \\ ${ }^{1}$ Department of Urology, National Cancer Center/National Clinical Research Center for Cancer/Cancer Hospital, Chinese Academy of Medical \\ Sciences and Peking Union Medical College, Beijing, China; ${ }^{2}$ Department of Urology, Peking University First Hospital, Institute of Urology, Peking \\ University, National Urological Cancer Center, Beijing, China \\ Contributions: (I) Conception and design: Q Zhao, F Yang, N Xing; (II) Administrative support: X Li, N Xing; (III) Provision of study materials or \\ patients: Q Zhao, X Li; (IV) Collection and assembly of data: Q Zhao, X Li; (V) Data analysis and interpretation: H Hao, L Wu; (VI) Manuscript \\ writing: All authors; (VII) Final approval of manuscript: All authors. \\ "These authors contributed equally to this work. \\ Correspondence to: Xuesong Li, MD, PhD. Department of Urology, Peking University First Hospital, Institute of Urology, Peking University, National \\ Urological Cancer Centre, No. 8 Xishiku St, Xicheng District, Beijing 100034, China. Email: pineneedle@sina.com; Nianzeng Xing, MD, PhD. \\ Department of Urology, National Cancer Center/National Clinical Research Center for Cancer/Cancer Hospital, Chinese Academy of Medical \\ Sciences and Peking Union Medical College, No. 17, Panjiayuan South Li, Chaoyang, Beijing 100021, China. Email: xingnianzeng@126.com.
}

Background: In recent years, the construction of orthotopic neobladder has become the focus of research. Our study is aim to illustrate and evaluate surgical techniques, oncologic and functional outcomes of two types of modified ileal orthotopic neobladders (IONB).

Methods: We retrospectively reviewed of 64 patients who underwent laparoscopic radical cystectomy (LRC) + Xing's neobladder ( $n=41)$, Institute of Urology Peking University neobladder (IUPUB) (n=23) at two tertiary referral centers. Then demographic characteristics, operation outcomes were analyzed. In addition, postoperative complication rates, oncologic outcomes, continence, and neobladders' function were further evaluated.

Results: All LRC + IONB were carried out in 64 patients successfully. Pathological results show that 63 were urothelial carcinoma and 1 was adenocarcinoma. Time to flatus was 3 [1-6] d in Xing's neobladder group while 3 [2-5] d in IUPUB group $(\mathrm{P}=0.049)$. In terms of overall survival (OS), cancer specific survival (CSS) and recurrence-free survival (RFS), there is no statistical difference between the two groups. Overall, grade 1-2 complications were $36 \%$, grade $3-4$ complications were $1.6 \%$ on the 30 th day, and $15.6 \%, 1.6 \%$ respectively on the 90th day. On the whole, daytime and night continence were $96.4 \%, 83.6 \%$ respectively one year after operation.

Conclusions: The two types of modified IONB are safe and feasible. The technical improvements significantly simplify the procedure and decrease the difficulty of the surgery, probably shorten the operative time and reduce blood loss. Furthermore, they take the advantage of less late complications and comparative good functional outcomes without affecting the prognosis of oncology. In addition, Xing's neobladder is more beneficial to the recovery of postoperative gastrointestinal function.

Keywords: Bladder cancer; ileal orthotopic neobladder; surgical techniques; oncologic outcomes; functional outcomes

Submitted Mar 22, 2021. Accepted for publication Jun 08, 2021.

doi: 10.21037/tau-21-251

View this article at: https://dx.doi.org/10.21037/tau-21-251 


\section{Introduction}

Radical cystectomy (RC) is a standard surgical method for the treatment of muscular invasive and high-risk nonmuscle invasive bladder cancer (1). Urinary diversion (UD) is an important part after RC. Since Lilien OM (2) first reported the clinical experience of orthotopic neobladder surgery in 1979, it has been rapidly developed and improved, and now it has become one of the standard urinary diversion methods after RC (3).

The orthotopic neobladder is a pouch synthesized by de-tubing and re-stitching the intestinal tube, which is characterized by low pressure, high capacity and good compliance (4). Ileum is an ideal and most widely used intestinal material for orthotopic neobladder at present (5). There are many kinds of IONB, among which Studer and Hautmann are the two most widely used (6-8). However, in practice, surgical procedures of Studer and Hautmann IONB are complicated, operation time is longer, and blood loss is large. These deficiencies suggest that we need to constantly improve and develop new IONB with relatively simple surgical techniques. Therefore, the study summarized the clinical features and surgical techniques of two types of modified IONB in two centers, and discussed their safety, feasibility, advantage and prognosis. In the meanwhile, we compared outcomes of the two neobladders via intracorporeal and extracorporeal approach. We present the following article in accordance with the STROBE reporting checklist (available at https://dx.doi.org/10.21037/ tau-21-251).

\section{Methods}

\section{Patients}

We retrospectively analyze a total of 64 patients with bladder cancer suitable for orthotopic neobladder surgery. Among them, 41 patients who underwent LRC with intracorporeal orthotopic neobladder (iONB) in Cancer Hospital, Chinese Academy of Medical Sciences from July 2013 to July 2019 were enrolled, abbreviated as Xing's neobladder group; 23 patients who underwent LRC with extracorporeal modified orthotopic neobladder (eONB) in Peking University first Hospital from August 2017 to October 2019 were included, abbreviated as Institute of Urology Peking University neobladder (IUPUB) group.

Inclusion criteria for IONB included that urethra was intact and the function of external sphincter was good, tumor margin of urethra was negative, no obvious intestinal disease, no distant tumor metastasis. Exclusion criteria included urethral tumor, urinary incontinence, urethral strictures, severe renal and hepatic dysfunction, severe intestinal disease and systemic comorbidities. All patients who underwent cystoscopy and biopsy or transurethral resection of bladder tumor (TURBT) were diagnosed with bladder cancer for LRC. All patients received pelvic enhanced computed tomography or magnetic resonance imaging and ultrasonography, bone scans, chest X-rays. Demographics, clinical, pathological, perioperative, and follow-up data were collected and evaluated.

The study was conducted in accordance with the Declaration of Helsinki (as revised in 2013). The study was approved by Research Ethics Committee of Cancer Hospital, Chinese Academy of Medical Sciences and Peking University first Hospital and informed consent was taken from all the patients.

\section{Surgical techniques}

\section{Patient positioning and trocar placement}

General anesthesia. All the patients were placed in the Trendelenburg position. Five trocars distribution was constructed in an "arch shape" as we previously reported $(9,10)$.

\section{LRC and pelvic lymph node dissection}

LRC and pelvic lymph node dissection (PLND) were performed according to standard laparoscopic procedure (11).

\section{Construction of the orthotopic neobladders Xing's orthotopic neobladder}

To construct the reservoir, a $60 \mathrm{~cm}$ ileum from about $25 \mathrm{~cm}$ proximal ileocecum was cut off under the illumination of another laparoscopic light system (Figure 1A). Then bowel continuity was restored using a stapled side-to-side anastomosis. The proximal $10 \mathrm{~cm}$ ileum was reversed to the right and anastomosed with the distal ileum as right isoperistaltic afferent limb of the neobladder (Figure 1B). The middle $40 \mathrm{~cm}$ of the ileum was detubularized with an ultrasonic scalpel along their antimesenteric line. The posterior wall of the neobladder was closed by continuous suturing of adjacent detubularized ileal walls using 3-0 Vicryl sutures (Figure 1C), the posterior wall of the neobladder was anastomosed with the posterior wall of the urethra (Figure 1D). Then, the catheter and two single $\mathrm{J}$ stents were introduced from the urethra. The ureters were spatulated for $2-3 \mathrm{~cm}$ and end-to-end 

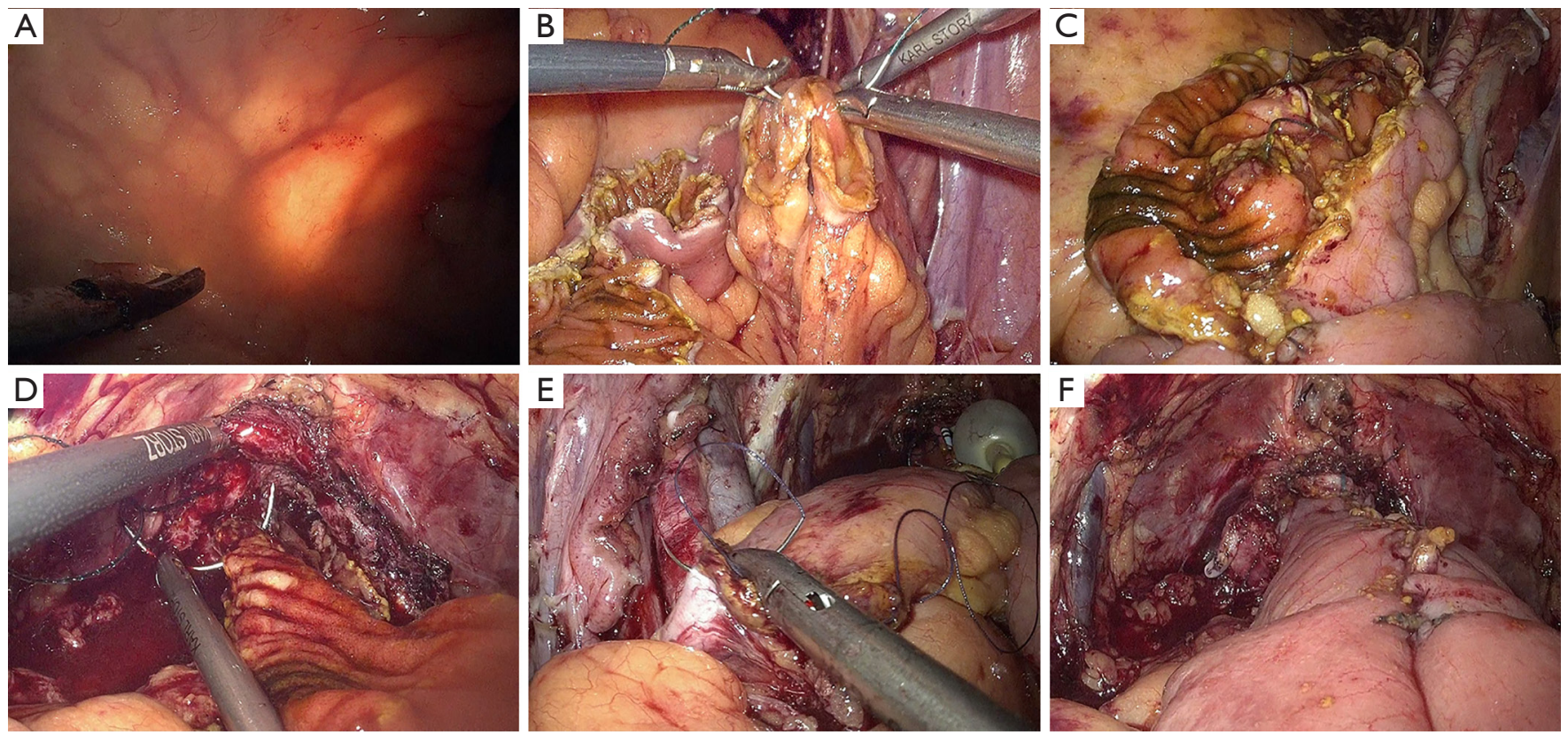

Figure 1 Surgical procedures for the construction of Xing's neobladder. (A) A $60 \mathrm{~cm}$ ileum from about $25 \mathrm{~cm}$ proximal ileocecum was cut off under the illumination of another laparoscopic light system; (B) the proximal $10 \mathrm{~cm}$ ileum was reversed to the right and anastomosed with the distal bowel as right isoperistaltic afferent limb of the neobladder; (C) the posterior wall of the neobladder was closed by continuous suturing of adjacent detubularized ileal walls using 3-0 Vicryl sutures; (D) the posterior wall of the neobladder was anastomosed with the posterior wall of the urethra; (E) the ureters were spatulated for 2-3 cm and were end-to-end anastomosed with ipsilateral limb in a continuous manner using 3-0 Vicryl sutures respectively; (F) the anterior wall of the neobladder was folded forward and the free edges were sutured to achieve a spherical configuration.

anastomosed with ipsilateral limb in a continuous manner using 3-0 Vicryl sutures respectively (Figure 1E). Finally, the anterior wall of the neobladder was folded forward and the free edges were sutured to achieve a spherical configuration (Figure $1 F$ ). Surgical animation video for surgical steps of Xing's neobladder can be viewed in Video 1. The specimens were extracted through an enlarged suprapubic port incision for male, and through the vagina for female.

\section{Institute of Urology Peking University neobladder (IUPUB)}

A $54-\mathrm{cm}$ ileal segment at approximately $25 \mathrm{~cm}$ proximal to the ileocecal valve was dissected. The ileal segment was marked at $12 \mathrm{~cm}, 24 \mathrm{~cm}$ and $39 \mathrm{~cm}$ with sutures, which indicated folding points and the beginning of the afferent limb, respectively (Figure $2 A$ ). The proximal $15 \mathrm{~cm}$ of the ileum was used for the afferent limb. The distal $39 \mathrm{~cm}$ of the ileum was detubularized along the antimesenteric border and folded at the mark points (Figure 2B). Suture the neighboring edges and the parallel edges respectively
(Figure 2C,D,E,F). A small opening was left for anastomosis with the urethra. The distal ureters were sutured into a $2.5-\mathrm{cm}$-long combined ureter, and anastomosed to the afferent limb. Two $7 \mathrm{~F}$ single-J stents were inserted in each of the two ureters and secured to a stoma catheter. Bladder cystostomy from a punctured incision was performed (Figure $2 F$ ). Then, the neobladder was put into the peritoneal cavity. The small opening of the neobladder and the urethra was anastomosed under laparoscope. Surgical animation video for surgical steps of IUPUB also can be viewed in Video 2.

\section{Postoperative care and follow-up}

The pelvic drain was extracted when the volume of drainage was $<100 \mathrm{~mL} /$ day in 3 consecutive days. The catheter was extracted when the cystography of the neobladder about 3 weeks postoperatively showed no leakage.

The patients were reexamined at 6 and 12 weeks after discharge, and thereafter every 3 months. Postoperative complications according to the Clavien-Dindo classification 

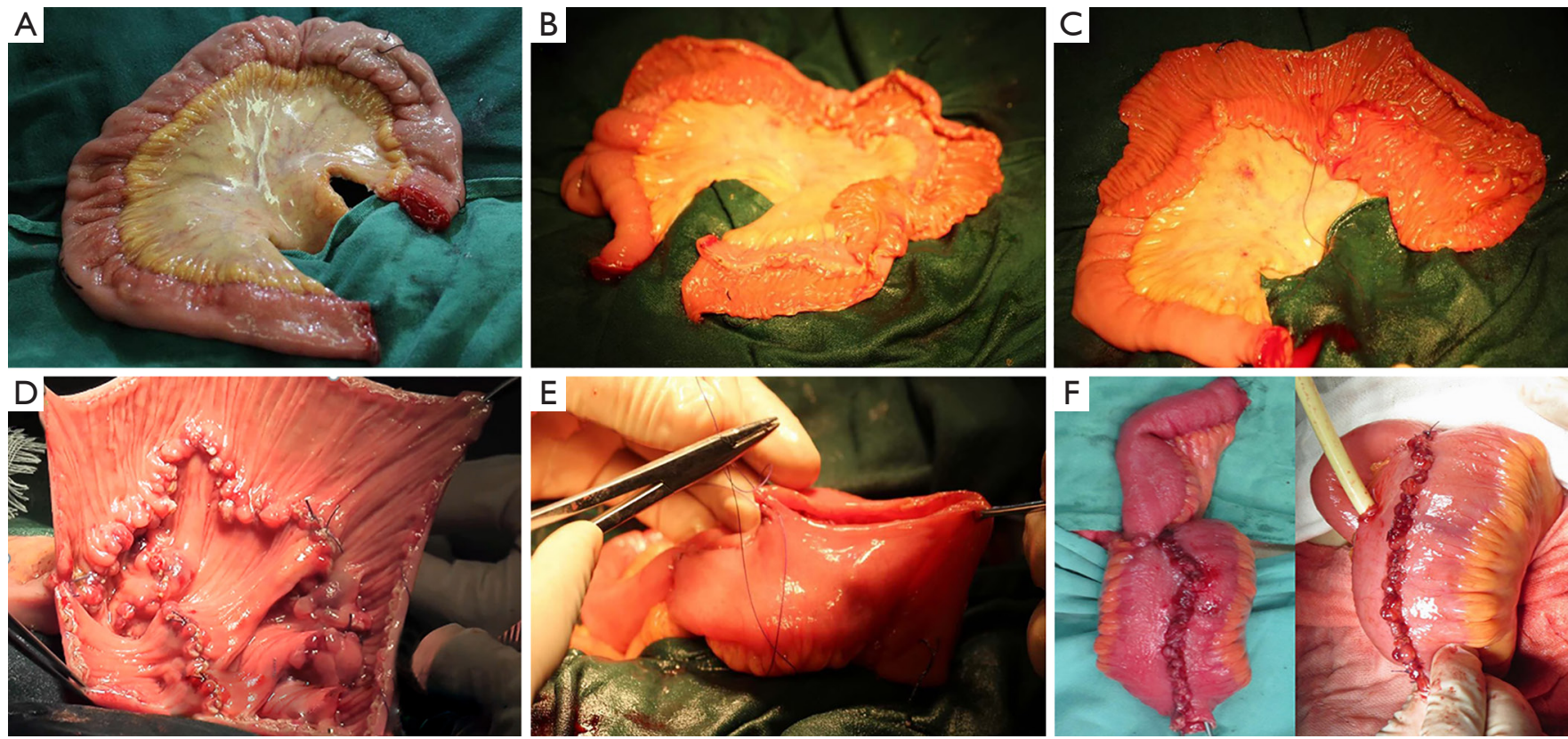

Figure 2 Surgical procedures for the construction of IUPUB. (A) The 54-cm ileal segment was marked at $12 \mathrm{~cm}, 24 \mathrm{~cm}$ and $39 \mathrm{~cm}$ with sutures, which indicated folding points and the beginning of the afferent limb, respectively; (B) the distal $39 \mathrm{~cm}$ of the ileum was detubularized along the antimesenteric border and folded at the mark points; (C) first fold of the distal ileal segment with the two 12 cm segments sutured together; (D) second fold and forming the posterior wall of the neobladder; (E) closing the anterior wall of the neobladder and leaving an opening to anastomose with the urethra; (F) completing the construction of the IUPUB.

at 30- and 90-day. Urinary continence (patients achieved total control or occasional dribbling or require 0 or 1 pad were considered continent; those who have normal physical activity requiring for more than 2 pads/day were defined as incontinence), neobladders' function and renal function were followed-up.

\section{Statistical analysis}

For continuous data, the variables in accordance with the normal distribution are expressed as mean \pm standard deviations (SD), and Student t test is used for the comparison between groups; the variables that do not accord with the normal distribution are expressed as the median, and Mann-Whitney test is used for the comparison between groups. For categorical variables, Median (range) were used to and compared with $\chi^{2}$ test. Survival probability within an event history analysis was calculated by KaplanMeier. A difference of $\mathrm{P}<0.05$ was deemed as statistically significant. The SPSS 24.0 statistical software was adopted for data analyses.

\section{Results}

As shown in Table 1 that there were no significant statistical differences in demographic and clinicopathologic characteristics between the two groups. Overall, the average age was $57.16 \pm 10.44$ years old, which showed the patients were relatively young; the median scores of CCI and ASA were 2, 2 respectively, which might indicate that most patients had a relatively good physical condition. In terms of pathological types, among 64 patients, 63 were urothelial carcinoma and 1 was adenocarcinoma. There were 38 patients with pathological stage $\geq \mathrm{T} 2$ and 26 patients with $\leq \mathrm{T} 1$, of which 26 cases all had a history of TURBT and postoperative BCG bladder instillation, and presented bladder cancer recurrence.

All LRC + IONB were carried out in 64 patients successfully. Operative outcomes were summarized in Table 2. Time to flatus was 3 [1-6] d in Xing's neobladder group while 3 [2-5] d in IUPUB group $(\mathrm{P}=0.049)$. No statistical difference was found in total operative time between the two groups. In Xing's neobladder group, the 
Table 1 Demographic and clinicopathologic characteristics of patients

\begin{tabular}{|c|c|c|c|c|}
\hline Variables & Overall & Xing's neobladder & IUPUB & $P$ \\
\hline Sex, $n$ & & & & 0.073 \\
\hline Male & 52 & 36 & 16 & \\
\hline Female & 12 & 5 & 7 & \\
\hline $\mathrm{BMI}\left(\right.$ mean $\pm \mathrm{SD}$ ), $\mathrm{kg} / \mathrm{m}^{2}$ & $24.73 \pm 3.66$ & $25.45 \pm 3.80$ & $23.48 \pm 3.08$ & 0.200 \\
\hline $\mathrm{CCl}$, median [range] & $2[2-6]$ & 2 [2-6] & $2[2-5]$ & 0.277 \\
\hline ASA score, median [range] & $2[1-4]$ & $2[1-4]$ & $2[1-3]$ & 0.416 \\
\hline Pathology type, n & & & & 0.641 \\
\hline Pathologic stage, $n$ & & & & 0.476 \\
\hline$\leq \mathrm{T} 1$ & 26 & 18 & 8 & \\
\hline$\geq \mathrm{T} 2$ & 38 & 23 & 15 & \\
\hline Pathologic grade, $\mathrm{n}$ & & & & 0.340 \\
\hline G1 & 5 & 4 & 1 & \\
\hline G2 & 5 & 3 & 2 & \\
\hline G3 & 44 & 30 & 14 & \\
\hline Unknown & 10 & 4 & 6 & \\
\hline
\end{tabular}

BMI, body mass index; ASA, American Society of Anesthesiologists; CCI, Charlson comorbidity index; TURBT, transurethral resection of bladder tumor.

time of urinary diversion was $177.38 \pm 43.63 \mathrm{~min}$, while the time of suturing pouch was $23.43 \pm 2.86 \mathrm{~min}$ in IUPUB group. As shown in Figure 3, the total operative time and time of urinary diversion had significant statistical differences between the first 20 cases and the last 21 cases in Xing's neobladder group who all underwent iNOB $(\mathrm{P}<0.05)$. There was a total of 4 patients with positive surgical margin, mainly due to the late stage of bladder cancer.

In terms of postoperative complications, as shown in Table 3, there were no significant difference between the two groups at 30-, 90-day. Overall, grade 1-2 complications were $36 \%$, grade $3-4$ complications were $1.6 \%$ on the 30 th day; and $15.6 \%, 1.6 \%$ respectively on the 90th day after operation. In Figure 4, we could find specifically that the early postoperative complications were mainly hypoproteinemia, followed by ileus and urinary tract infection. Metabolic acidosis was considered to be the most common complication at 90 -day after operation,
(4, 6\%); followed by ileus $(3,5 \%)$ and urinary tract infection (3, 5\%). Of the 64 patients, 2 were re-admitted owing to postoperative ileus, 1 was re-operated owing to postoperative peri-anastomotic bleeding, and 1 was admitted to ICU because of relatively poor cardiopulmonary function.

In terms of postoperative oncologic outcomes, 64 patients were followed up successfully, took the last followup time as the cutoff point, in the Xing's neobladder group, the median follow-up was 37.0 months, there were 1 cases of recurrence and 6 cases of metastasis. In addition, a total of 11 patients died, of which 6 died of bladder cancer and the remaining 5 died of other causes, the overall survival (OS), cancer specific survival (CSS) and recurrence-free survival (RFS) was $73.2 \%, 83.3 \%$, and $85.4 \%$, respectively. In IUPUB group, the median follow-up was 22.5 months, there were 2 cases of recurrence and 1 cases of metastasis. Besides, one patient died of bladder cancer and one died of 
Table 2 Operation outcomes

\begin{tabular}{|c|c|c|c|c|}
\hline Variables & Overall & Xing's neobladder & IUPUB & $\mathrm{P}$ \\
\hline $\begin{array}{l}\text { Time of urinary diversion } \\
\text { (mean } \pm \text { SD), min }\end{array}$ & - & $177.38 \pm 43.63$ & - & - \\
\hline Time of suturing pouch (mean \pm SD), min & - & - & $23.43 \pm 2.86$ & - \\
\hline Time of ambulation (d), median [range] & $1[1-4]$ & $1[1-4]$ & $1[1-4]$ & 0.689 \\
\hline${ }^{*}$ Time to liquid diet (d), median [range] & $4[1-10]$ & $3.5[1-8]$ & $4[2-10]$ & 0.504 \\
\hline Time to pelvic drain removal $(d),($ mean $\pm S D), d$ & $9.77 \pm 4.77$ & $10.1 \pm 4.47$ & $9.17 \pm 5.31$ & 0.846 \\
\hline Lymph node yield (mean $\pm \mathrm{SD}$ ), $\mathrm{n}$ & $16.87 \pm 8.49$ & $18.76 \pm 7.89$ & $13.52 \pm 8.65$ & 0.938 \\
\hline Lymph node positive, median [range], $\mathrm{n}$ & $0[0-27]$ & $0[0-18]$ & $0[0-27]$ & 0.226 \\
\hline Positive surgical margin, $n$ & 4 & 3 & 1 & - \\
\hline
\end{tabular}

PLND, pelvic lymph node dissection; EBL, estimated blood loss. *exclude cases of ileus.

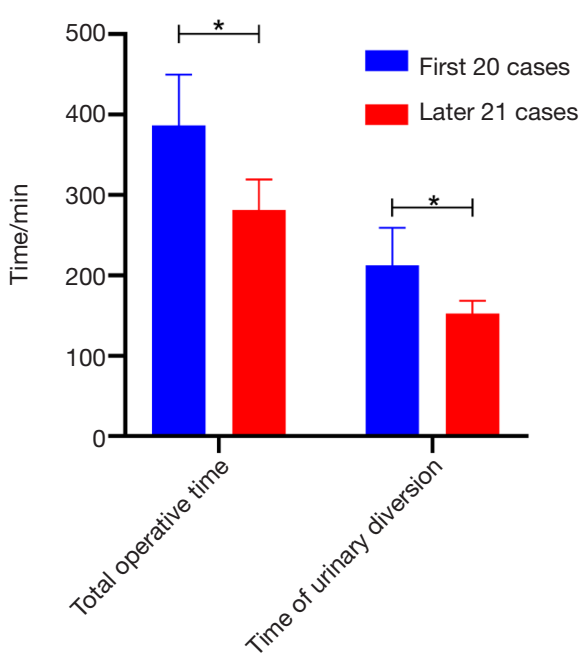

Figure 3 perative time of the laparoscopic radical cystectomy with intra-corporeal orthotopic neobladder. The total operative time and time of urinary diversion had been significantly reduced between the first 20 cases and the last 21 cases. ${ }^{*} \mathrm{P}<0.05$.

myocardial infarction (Table 4). In terms of OS, CSS, RFS, it could be seen from Figure 5 that there were no statistical differences between the two groups.
As shown in Table 5 that no statistical differences were found in daytime and night continence between the two groups. Overall, daytime and night continence were $96.4 \%$, $83.6 \%$ respectively one year after operation. There were no significant differences in bladder capacity, residual volume, max flow rate between the two groups up to the last followup time. Overall, the median capacity, residual volume, max flow rate of the neobladder is $400(150-750) \mathrm{mL}, 0.0$ $(0-50) \mathrm{mL}, 20.6(6.9-39.5) \mathrm{mL} / \mathrm{sec}$. In addition, none of the patients underwent clean intermittent catheterization. In the Xing's neobladder group, the preoperative serum creatinine was $81.25 \pm 18.34 \mu \mathrm{mol} / \mathrm{L}$, postoperative was $81.81 \pm 20.18$ $\mu \mathrm{mol} / \mathrm{L}$; while $81.81 \pm 20.18 \mu \mathrm{mol} / \mathrm{L}, 81.52 \pm 24.40 \mu \mathrm{mol} / \mathrm{L}$ respectively in IUPUB group. Furthermore, we found that there was no statistical difference in creatinine between the two groups, also no significant difference in creatinine before and after operation in each group.

\section{Discussion}

The two types of modified INOB have obtained good results, the operation is safe and feasible, and the postoperative oncologic and functional outcomes are also encouraging. 
Table 3 Postoperative complication rates

\begin{tabular}{lccc}
\hline Variables & Overall & Xing's neobladder & IUPUB \\
\hline 30-day complication, n (\%) & & & \\
Minor (I-II) & $23 / 64(36.0)$ & $14 / 41(34.1)$ & $9 / 23(39.1)$ \\
Major (III-V) & $1 / 64(1.6)$ & $1 / 41(2.4)$ & $0 / 23(0.0)$ \\
90-day complication, n (\%) & & $5 / 41(12.2)$ & $5 / 23(21.7)$ \\
Minor (I-II) & $10 / 64(15.6)$ & $0.571(0.0)$ & $1 / 23(4.3)$ \\
Major (III-V) & $1 / 64(1.6)$ & $37.0(8.5-82.0)$ & $22.5(3.8-34.1)$ \\
Follow-up median (range), month & $31.6(3.8-82.0)$ & & $<0.001$ \\
\hline
\end{tabular}

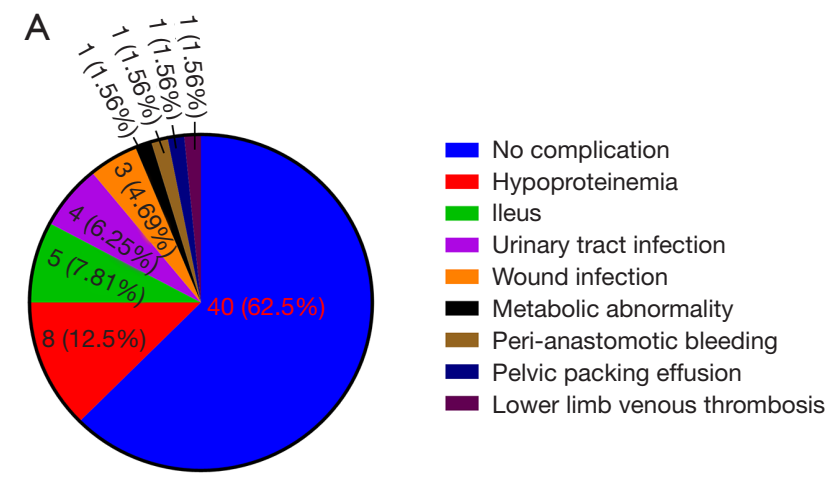

30-day complication rates
B

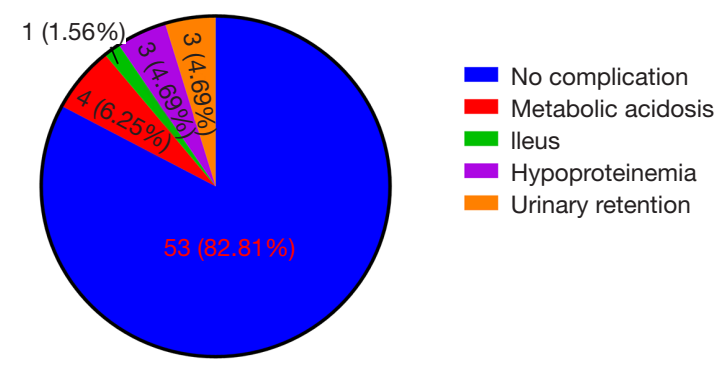

90-day complication rates

Figure 4 The specific postoperative complications at 30- and 90-day. (A) The mainly postoperative complication at 30-day was hypoproteinemia, which occurred in 8 cases (12\%), followed by ileus $(5,8 \%)$ and urinary tract infection (4, 6\%). (B) Metabolic acidosis was considered to be the most common complication at 90 -day after operation, 4 cases (6\%), followed by ileus (3, $5 \%)$ and urinary tract infection $(3,5 \%)$.

Table 4 Oncologic outcomes

\begin{tabular}{lcccc}
\hline Variables & Overall & Xing's neobladder & IUPUB & P \\
\hline Recurrence, $\mathrm{n}(\%)$ & $3 / 64(4.7 \%)$ & $1 / 41(2.4 \%)$ & $2 / 23(8.7 \%)$ & $1 / 23(4.3 \%)$ \\
Metastases, $\mathrm{n}(\%)$ & $7 / 64(10.9 \%)$ & $6 / 41(14.6 \%)$ & $91.3 \%$ & 0.290 \\
Overall Survival & $79.7 \%$ & $73.2 \%$ & $95.5 \%$ & 0.363 \\
Cancer specific survival & $87.9 \%$ & $83.3 \%$ & $87.0 \%$ & 0.950 \\
Recurrence-free survival & $85.9 \%$ & $85.4 \%$ & $22.5(3.8-34.1)$ & $<0.001$ \\
Follow-up median (range), month & $31.6(3.8-82.0)$ & $37.0(8.5-82.0)$ & &
\end{tabular}

Compared with ileal conduit, orthotopic neobladder was beneficial to patients in terms of autonomous urination without abdominal ostomy, which maintained a good personal image, improved health related quality of life $(12,13)$. At present, ileal orthotopic neobladder can be considered for patients who are suitable for RC after excluding the contraindications of ileal orthotopic neobladder (14). The ideal orthotopic neobladder should 

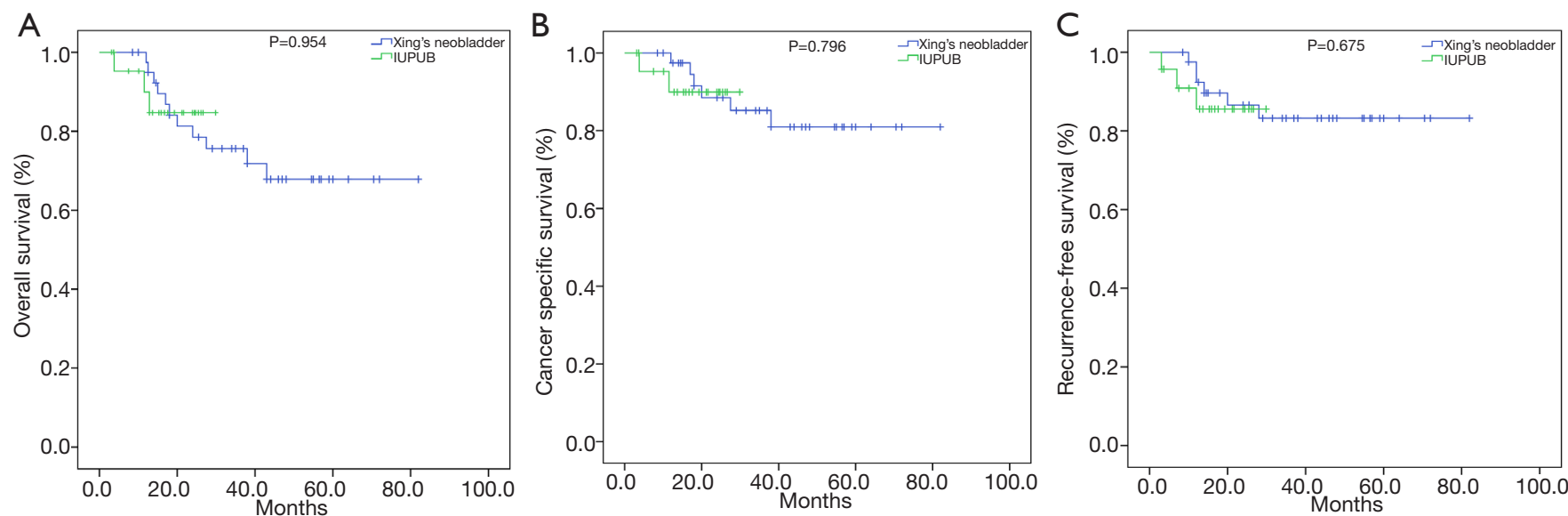

Figure 5 Kaplan-Meier Estimates between Xing's neobladder and IUPUB group. (A) Figure shown are data for overall survival (OS), there was no statistical difference between the two groups $(\mathrm{P}=0.290)$. (B) Figure shown are data for cancer specific survival (CSS), there was no statistical difference between the two groups $(\mathrm{P}=0.363)$. (C) Figure shown are data for recurrence-free survival (RFS), there was no statistical difference between the two groups $(\mathrm{P}=0.950)$.

Table 5 Continence, neobladder function and creatinine outcomes

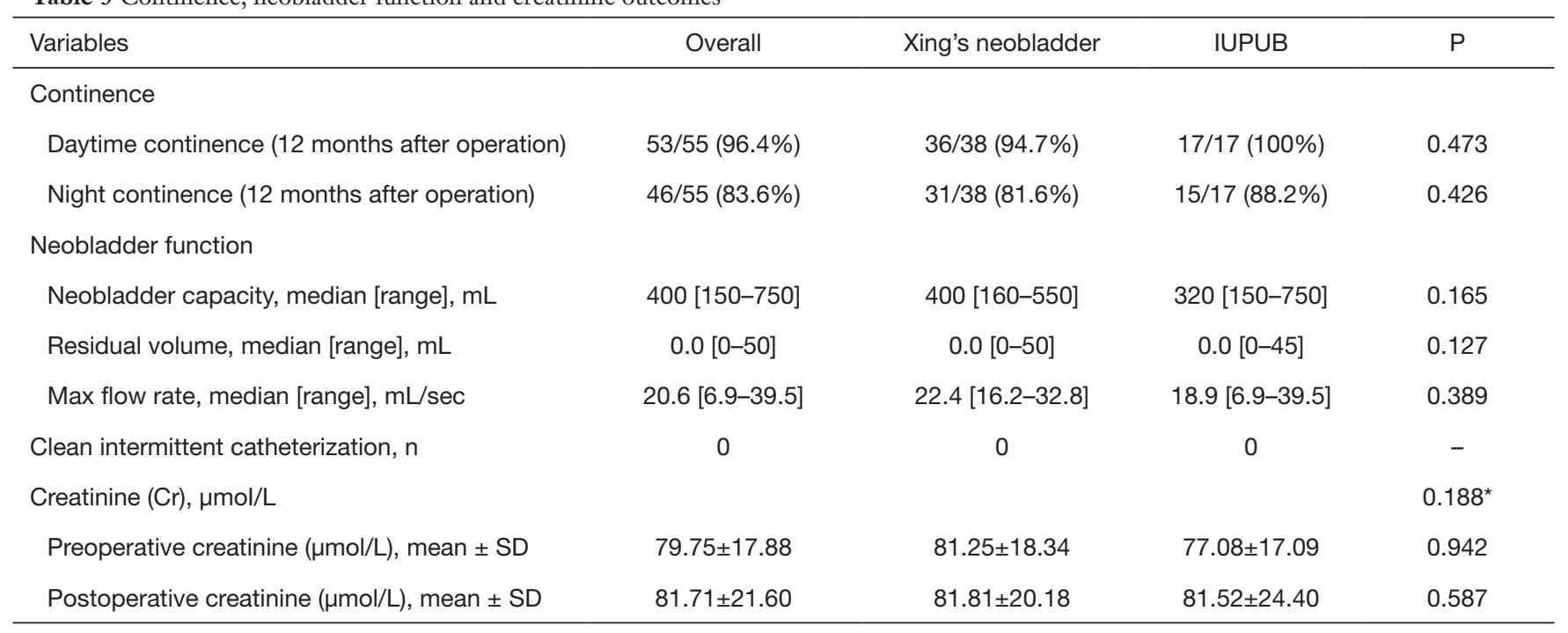

${ }^{*} P$ value was calculated by paired $T$ test.

have the following characteristics $(15,16)$ : (I) having physiological excretory function of the human body; (II) storing urine under low pressure; (III) having sufficient capacity and good compliance; (IV) no water and electrolyte imbalance; (V) no ureteral reflux and impairment of renal function; (VI) simple and easy to operate. The Studer neobladder uses a non-detubularized isoperistaltic ileum as an input loop to prevent urine reflux (17). Compared with the Studer neobladder, the two modified neobladders also use this method, but Xing's neobladder adopted bilateral isoperistaltic afferent limbs (10). Schoöndorf D reviewed and analyzed 64 cases of anastomotic stenosis of unilateral or bilateral ureteral ileal input limbs of Studer neobladder. It was found that the stenosis rate of left ureteral anastomosis was two times higher than that of right, which was probable related to the impairment of blood supply and long-term chronic ischemia of left ureter (18). However, the advantage of Xing's neobladder was that the left ureter needn't be excessively dissociated during the operation, and could be directly anastomosed with the input limb, which 
could better protect the blood supply of the left ureter and avoid effectively anastomotic stricture. In addition, the two neobladders simplified the way of folding and suturing, improved the feasibility of the operation. Up to now, there was no urinary retention caused by torsion between neobladder and urethral anastomosis during the follow-up of these 64 patients.

Desai MM (19) reported 132 cases of robot-assisted radical cystectomy (RARC) + iONB, the result showed that the mean operation time was 7.6 hours (range 4.4 to 13 ), blood loss was $430 \mathrm{~mL}$ (range 50 to 2,200). Simone G (20) reported 70 cases of RARC with construction of ileal neobladder. The median operation time was 420.0 (265-760) min, blood loss was $500.0(100-2,200) \mathrm{mL}$. In our study, the operative time and blood loss were $328.55 \pm 68.94 \mathrm{~min}, 125(20-700) \mathrm{mL}$ respectively. We could find that our neobladders shorten the operation time, reduce blood loss, which is worthy of clinical promotion to a certain extent. Gill IS (21) first reported LRC $+\mathrm{iONB}$ in 2002. With the development of laparoscopy and robot-assisted technology, there are more and more reports about iONB (22-24). Collins JW (25) studied the learning curve of RARC and iONB performed by two physicians with different qualifications, and evaluated the learning curve. Finally, it was concluded that under the guidance of experienced superior doctors for young doctors, the operation time and complications of the latter 10 cases was significantly reduced than that of the initial 10 cases $(\mathrm{P}<0.01)$. Our results showed that there were significant differences in total operation time, neobladder construction time between the initial 20 patients and the latter 21 patients who underwent LRC $+\mathrm{iONB}$ in Xing's neobladder group. Therefore, we conclude that with the accumulation of surgical experience, the optimization of surgical procedures, the learning curve of iONB will be greatly shortened. In addition, we summed up the following experience about iONB: (I) we creatively used another set of endoscopic light source system to irradiate the mesentery on the back so that mesenteric artery could be seen clearly, which contributed to preserving mesenteric vessels. (II) When suturing the pouch, our method was to use 3-0 absorbable thread to stitch 4-5 stitches intermittently per $20 \mathrm{~cm}$ intestinal tube, then lift both ends of the suture by the assistant to make it have a certain tension, and then use 3-0 barbed thread to suture continuously and tighten the suture when 4-5 stitches were completed, which could not only close tightly, but also save operation time. (III) When the neobladder was anastomosed with the urethra, after excluding the male patients with prostate cancer before operation, part of the capsule of the tip of prostate could be preserved and directly anastomosed with the neobladder. This not only reduced the difficulty of anastomosis, but also was more beneficial to preserving the external sphincter and vascular nerve bundle of the urethra. (IV) The surgical specimens could be extracted transvaginal for female patient, which could avoid incision-related complications and was more cosmetic.

In terms of postoperative oncologic outcomes, Desai MM (19) reported mid-term oncologic outcomes of RARC with iONB. The study included 132 patients, mean follow-up was 2.1 years (range 0.1 to 9.8). The results found five-year OS, CSS and RFS were $72 \%, 72 \%$ and $71 \%$, respectively, which was lower than the results of our research in Xing's neobladder. Simone (20) did similar research and found two-year OS, CSS, RFS were $82.4 \%$, $82.4 \%, 72.5 \%$ respectively, which was lower than the results of our research in IUPUB. Simone et al. (26) also made a retrospective comparative analysis of the oncologic results of 64 cases of RARC with iONB and 299 cases of RARC with eONB. The results indicated that RARC with iONB provided comparable oncologic outcomes to eONB. In our study, we found that there were no differences in terms of OS, CSS, RFS between the two groups and both obtained good oncologic outcomes. However, longer follow-up was needed to further confirm.

In terms of the functional outcomes of the neobladders, Studer UE (27) reported their 20-year surgical experience of orthotopic ileal neobladder. The daytime and night continence one year after operation were $92 \%$ and $79 \%$, respectively. In another three-center study of 104 cases, the median follow-up was 88 months, the daytime continence was $98 \%$, and the night continence was $76 \%$ (28). Hautmann RE (29) reported the surgical experience of 67 cases of Hautmann orthotopic neobladder. The daytime and night continence rate were $95 \%$ and $73 \%$ respectively one year after operation, and the median bladder functional capacity was $450 \mathrm{~mL}$, which was similar to that of normal bladder. Satkunasivam (30) retrospectively analyzed the functional results of 28 cases of RARC with iONB and 79 cases of RARC with eONB. They concluded that iONB had comparable urodynamic features and quality-of-life outcomes to eONB. However, the daytime continence was worse for iONB, which was mainly attributed to the short follow-up period. In our study, the one-year daytime and night continence were $96.4 \%, 83.6 \%$ respectively, which were basically similar to the results reported above. 
In terms of complications, a multicenter study (31) found that the most common complication of RARC + UD was gastrointestinal related complications, followed by infection and genitourinary system related complications. Compared with $23 \%$ of gastrointestinal complications in $\mathrm{RARC}+\mathrm{eONB}$, the gastrointestinal-related complications of RARC + iONB were only $10 \%(\mathrm{P}<0.001)$. The main reason was attributed to that $\mathrm{iONB}$ can reduce the loss of gastrointestinal fluid and intestinal wall edema caused by excessive traction and movement in vitro. In our study, although there were no statistical difference in the complications between the two groups, Xing's neobladder was more beneficial to the recovery of postoperative gastrointestinal function. Hautmann RE (6) reported postoperative complications in 1,013 patients and showed that the 90-day complication rate of Clavien grade $\geq 1$ was $58 \%$. Besides, the most common complications were infection, urinary system complications, digestive system complications. Studer UE (27) retrospectively analyzed 482 patients with orthotopic neobladder and found that early postoperative complication ( $<30$ days) rate was $32.8 \%$, mainly including acute pyelonephritis, ileus and pulmonary embolism; and long-term complication rate was $54.9 \%$, mainly including ileus, urinary retention and metabolic complications. However, only cystectomy-related and diversion-related complications were counted in this study, and other types of complications were not counted.

In our study, postoperative 30-day complications were $37.6 \%$, mainly a complication of hypoproteinemia, but and cystectomy-related and diversion-related complications only were $12.0 \%$. The most common postoperative complication was metabolic disorder, 4 patients developed metabolic acidosis in varying degrees with no related symptoms of metabolic acidosis. PH value returned to normal after oral administration of sodium bicarbonate. A study (32) shown that metabolic acidosis affects the recovery of urinary continence in the early postoperative period. Therefore, it is very necessary to monitor timely the change of $\mathrm{PH}$ value in the body. Compared with the results of the literature, our two types of neobladders had a low incidence of late postoperative complications, which were mainly attributed to our special postoperative follow-up and management. Concretely, neobladder irrigation was performed on the first day after operation (100-150 mL saline every 2 hours). Cystography was performed three weeks postoperatively, urodynamic examination was performed 6 months after operation, and patients' continence and quality of life were followed up by an independent follow-up team. We have made a patient's education manual to guide patients' postoperative precautions and rehabilitation training. In addition, we set up a follow-up management clinic of neobladder, which took the advantage that we could not only give some health education to patients, such as postoperative nursing methods, matters needing attention, postoperative rehabilitation exercise, but also deal with the complications of patients in time.

This study inevitably has some limitations. First of all, this study is a retrospective study, which leads to certain selective bias. Second, the numbers of cases in this study are small and the follow-up time is relatively short.

\section{Conclusions}

In summary, the two types of modified IONB are safe and feasible. The technical improvements significantly simplify the procedure and decrease difficulty of the surgery, probably shorten the operative time and reduce blood loss. Furthermore, they take the advantage of less late complications and comparative functional outcomes without affecting the prognosis of oncology. In addition, Xing's neobladder is more beneficial to the recovery of postoperative gastrointestinal function. However, a large sample, multicenter, prospective randomized controlled study is needed to further confirm its long-term prognosis.

\section{Acknowledgments}

We would like to thank foreign teacher Christopher Ott from English Academy, Peking Union Medical College for his help in polishing our paper.

Funding: This study was financially supported by the Capital Science and Technology Leading Talent Project (Project number: Z181100006318007) and Beijing Hope Run Special Fund of Cancer Foundation of China (Project number: LC2019B02).

\section{Footnote}

Reporting Checklist: The authors have completed the STROBE reporting checklist. Available at https://dx.doi. org/10.21037/tau-21-251

Data Sharing Statement: available at https://dx.doi. org/10.21037/tau-21-251

Conflicts of Interest: All authors have completed the ICMJE 
uniform disclosure form (available at https://dx.doi. org/10.21037/tau-21-251). Xuesong Li serves as an unpaid editorial board member of Translational Andrology and Urology. Nianzeng Xing serves as an unpaid editorial board member of Translational Andrology and Urology from Mar 2020 to Feb 2022. The other authors have no conflicts of interest to declare.

Ethical Statement: The authors are accountable for all aspects of the work in ensuring that questions related to the accuracy or integrity of any part of the work are appropriately investigated and resolved. The study was conducted in accordance with the Declaration of Helsinki (as revised in 2013). The study was approved by Research Ethics Committee of Cancer Hospital, Chinese Academy of Medical Sciences and Peking University first Hospital and informed consent was taken from all the patients.

Open Access Statement: This is an Open Access article distributed in accordance with the Creative Commons Attribution-NonCommercial-NoDerivs 4.0 International License (CC BY-NC-ND 4.0), which permits the noncommercial replication and distribution of the article with the strict proviso that no changes or edits are made and the original work is properly cited (including links to both the formal publication through the relevant DOI and the license). See: https://creativecommons.org/licenses/by-nc-nd/4.0/.

\section{References}

1. Gakis G, Efstathiou J, Lerner SP, et al. ICUD-EAU International Consultation on Bladder Cancer 2012: Radical cystectomy and bladder preservation for muscleinvasive urothelial carcinoma of the bladder. Eur Urol 2013;63:45-57.

2. Lilien OM, Camey M, et al. 25-Year Experience with Replacement of the Human Bladder (Camey Procedure). J Urol 2017;197:S173-9.

3. Meyer JP, Fawcett D, Gillatt D, et al. Orthotopic neobladder reconstruction--what are the options? BJU Int 2005;96:493-7.

4. Cacciamani GE, Rajarubendra N, Artibani W, et al. Robotic intracorporeal urinary diversion: state of the art. Curr Opin Urol 2019;29:293-300.

5. Hautmann RE. Urinary diversion highlights. Eur Urol 2006;50:1139-41.

6. Hautmann RE, de Petriconi RC, Volkmer BG, et al. Lessons learned from 1,000 neobladders: the 90-day complication rate. J Urol 2010;184:990-4; quiz 1235.

7. Daneshmand S. Orthotopic urinary diversion. Curr Opin Urol 2015;25:545-9.

8. Bianchi G, Sighinolfi MC, Pirola GM, et al. Studer Orthotopic Neobladder: A Modified Surgical Technique. Urology 2016;88:222-5.

9. Hong P, Ding GP, Hao H, et al. Laparoscopic Radical Cystectomy With Extracorporeal Neobladder: Our Initial Experience. Urology 2019;124:286-91.

10. Xing NZ, Kang N, Song LM, et al. Laparoscopic radical cystectomy with novel orthotopic neobladder with bilateral isoperistaltic afferent limbs: initial experience. Int Braz J Urol 2017;43:57-66.

11. Desai MM, Berger AK, Brandina RR, et al. Robotic and laparoscopic high extended pelvic lymph node dissection during radical cystectomy: technique and outcomes. Eur Urol 2012;61:350-5.

12. Cerruto MA, D'Elia C, Siracusano S, et al. Systematic review and meta-analysis of non RCT's on health related quality of life after radical cystectomy using validated questionnaires: Better results with orthotopic neobladder versus ileal conduit. Eur J Surg Oncol 2016;42:343-60.

13. Lee RK, Abol-Enein H, Artibani W, et al. Urinary diversion after radical cystectomy for bladder cancer: options, patient selection, and outcomes. BJU Int 2014;113:11-23.

14. Ong K, Herdiman O, Johnson L, et al. Orthotopic bladder substitution (neobladder): part I: indications, patient selection, preoperative education, and counseling. J Wound Ostomy Continence Nurs 2013;40:73-82.

15. Thurairaja R, Burkhard FC, Studer UE, et al. The orthotopic neobladder. BJU Int 2008;102:1307-13.

16. Chang DT, Lawrentschuk N, et al. Orthotopic neobladder reconstruction. Urol Ann 2015;7:1-7.

17. Studer UE, Varol C, Danuser H, et al. Orthotopic ileal neobladder. BJU Int 2004;93:183-93.

18. Schöndorf D, Meierhans-Ruf S, Kiss B, et al. Ureteroileal strictures after urinary diversion with an ileal segment-is there a place for endourological treatment at all? J Urol 2013;190:585-90.

19. Desai MM, Gill IS, de Castro Abreu AL, et al. Robotic intracorporeal orthotopic neobladder during radical cystectomy in 132 patients. J Urol 2014;192:1734-40.

20. Simone G, Papalia R, Misuraca L, et al. Robotic Intracorporeal Padua Ileal Bladder: Surgical Technique, Perioperative, Oncologic and Functional Outcomes. Eur Urol 2018;73:934-40.

21. Gill IS, Kaouk JH, Meraney AM, et al. Laparoscopic 
radical cystectomy and continent orthotopic ileal neobladder performed completely intracorporeally: the initial experience. J Urol 2002;168:13-8.

22. Beecken WD, Wolfram M, Engl T, et al. Robotic-assisted laparoscopic radical cystectomy and intra-abdominal formation of an orthotopic ileal neobladder. Eur Urol 2003;44:337-9.

23. Azzouni FS, Din R, Rehman S, et al. The first 100 consecutive, robot-assisted, intracorporeal ileal conduits: evolution of technique and 90-day outcomes. Eur Urol 2013;63:637-43

24. Jonsson MN, Adding LC, Hosseini A, et al. Robot-assisted radical cystectomy with intracorporeal urinary diversion in patients with transitional cell carcinoma of the bladder. Eur Urol 2011;60:1066-73.

25. Collins JW, Tyritzis S, Nyberg T, et al. Robot-assisted radical cystectomy (RARC) with intracorporeal neobladder - what is the effect of the learning curve on outcomes? BJU Int 2014;113:100-7.

26. Simone G, Tuderti G, Misuraca L, et al. Perioperative and mid-term oncologic outcomes of robotic assisted radical cystectomy with totally intracorporeal neobladder: Results of a propensity score matched comparison with open cohort from a single-centre series. Eur J Surg Oncol 2018;44:1432-8.

Cite this article as: Zhao Q, Yang F, Hao H, Li X, Wu L, Li X, Xing N. Surgical techniques, oncologic and functional outcomes of two types of modified ileal orthotopic neobladders. Transl Androl Urol 2021;10(7):2970-2981. doi: 10.21037/tau-21251
27. Studer UE, Burkhard FC, Schumacher M, et al. Twenty years experience with an ileal orthotopic low pressure bladder substitute--lessons to be learned. J Urol 2006;176:161-6.

28. Meyer JP, Blick C, Arumainayagam N, et al. A threecentre experience of orthotopic neobladder reconstruction after radical cystectomy: revisiting the initial experience, and results in 104 patients. BJU Int 2009;103:680-3.

29. Hautmann RE, de Petriconi R, Gottfried HW, et al. The ileal neobladder: complications and functional results in 363 patients after 11 years of followup. J Urol 1999;161:422-7; discussion 427-8.

30. Satkunasivam R, Santomauro M, Chopra S, et al. Robotic Intracorporeal Orthotopic Neobladder: Urodynamic Outcomes, Urinary Function, and Health-related Quality of Life. Eur Urol 2016;69:247-53.

31. Ahmed K, Khan SA, Hayn MH, et al. Analysis of intracorporeal compared with extracorporeal urinary diversion after robot-assisted radical cystectomy: results from the International Robotic Cystectomy Consortium. Eur Urol 2014;65:340-7.

32. Müller G, Butea-Bocu M, Brock O, et al. Association between Development of Metabolic Acidosis and Improvement of Urinary Continence after Ileal Neobladder Creation. J Urol 2020;203:585-90. 